\title{
Association between soluble forms of the receptor for advanced glycation end products and periodontal disease: a retrospective study
}

\author{
Keun-Suh Kim ${ }^{1}$, Yun Jong Lee ${ }^{2}$, Soyeon Ahn ${ }^{3}$, Yoon-Seok Chang ${ }^{3,4}$, Yonghoon Choi ${ }^{5}$, Hyo-Jung Lee \\ ${ }^{1}$ Department of Periodontology, Section of Dentistry, ${ }^{2}$ Division of Rheumatology, Department of Internal Medicine, \\ ${ }^{3}$ Division of Statistics, Medical Research Collaborating Center, ${ }^{4}$ Division of Allergy and Clinical Immunology, Department of Internal \\ Medicine, ${ }^{5}$ Department of Conservative Dentistry, Section of Dentistry, Seoul National University Bundang Hospital, Seongnam, Korea
}

\begin{abstract}
J Korean Assoc Oral Maxillofac Surg 2021;47:445-453)
Objectives: Periodontitis is the most common chronic disease that causes tooth loss and is related to systemic diseases such as cardiovascular disease and diabetes. An objective indicator of the current activity of periodontitis is necessary. Soluble forms of the receptor for advanced glycation end products (sRAGE) are markers that reflect the status of inflammatory diseases. In this study, the relationship between sRAGE and periodontitis was analyzed to determine whether it can be used to diagnose the current state of periodontitis.

Patients and Methods: Eighty-four patients without any systemic diseases were diagnosed with periodontitis using three classifications of periodontitis. Demographics and oral examination data such as plaque index (PI), bleeding on probing (BOP) index, and probing pocket depth (PPD) were analyzed according to each classification. In addition, correlation and partial correlation between sRAGE and the values indicating periodontitis were analyzed.

Results: In each classification, the level of sRAGE tended to decrease if periodontitis was present or severe, but this change was not statistically significant. sRAGE and periodontitis-related variables exhibited a weak correlation, among which the BOP index showed a relatively strong negative correlation $(\rho=0.20$ ). Based on this, on analyzing the correlation between the BOP index and sRAGE in the group with more severe periodontitis (PPD $\geq 5$ mm group, severe group of AAP/CDC [American Academy of Periodontology/Centers for Disease Control and Prevention], periodontitis group of López), the correlation further increased $(\rho=0.23,-0.40,-0.50)$. Partial correlation analysis of the sRAGE and BOP index showed a stronger negative correlation ( $\rho=0.36,-0.55,-0.45)$.

Conclusion: sRAGE demonstrated a tendency to decrease upon increased severity of periodontitis according to the classifications used. Above all, the correlation with the BOP index, which reflects the current state of periodontitis, was higher in the group with severe periodontitis. This indicates that the current status of periodontitis can be diagnosed through sRAGE.
\end{abstract}

Key words: Receptor for advanced glycation end products, Periodontal index, Periodontitis, Bleeding on probing index

[paper submitted 2021. 10. 28 / revised 2021. 11. 18 / accepted 2021. 11. 24]

\section{Introduction}

Periodontitis is a common chronic inflammatory disease. Reportedly, 796 million people worldwide have severe periodontitis $^{1}$, and $42 \%$ of 30 -year-old adults in the United

\footnotetext{
Hyo-Jung Lee

Department of Periodontology, Section of Dentistry, Seoul National University Bundang Hospital, 82 Gumi-ro 173beon-gil, Bundang-gu, Seongnam 13620, Korea

TEL: +82-31-787-7547

E-mail:periolee@gmail.com

ORCID: https://orcid.org/0000-0002-0439-7389
}

(c) This is an open-access article distributed under the terms of the Creative Commons Attribution Non-Commercial License (http://creativecommons.org/ licenses/by-nc/4.0/), which permits unrestricted non-commercial use, distribution, and reproduction in any medium, provided the original work is properly cited.

Copyright (C) 2021 The Korean Association of Oral and Maxillofacial Surgeons.
States $^{2}$ and more than $30 \%$ of adults in Korea are reported to suffer from periodontitis ${ }^{3}$. The loss of teeth due to periodontitis causes the loss of mastication function, leading to a poor quality of life ${ }^{4}$. In addition, the association of periodontitis with systemic diseases such as cardiovascular diseases and diabetes, as a cause of systemic inflammation due to bacteremia, is also attracting attention ${ }^{5}$.

Several modalities exist for the diagnosis of periodontitis. The most basic diagnostic process is the evaluation of alveolar bone loss through panoramic or periapical radiography. Subsequently, the dentist screens for redness of the gingival tissue and presence of swelling, edema, and abscesses through an intraoral examination ${ }^{6}$.

The loss of alveolar bone reflects the progression of periodontitis and does not necessarily indicate the current state 
of inflammation. In some patients, despite extensive alveolar bone loss, healthy gingiva is observed without severe inflammation after gingival recession. The state of periodontitis can also be verified through probing. A deep periodontal pocket with bleeding on probing (BOP) can be an important indicator of the active state of the existing periodontal lesion. However, the use of anti-thrombotic agents and presence systemic disease may cause a predisposition to bleeding, with smoking affecting the pathological bleeding tendency of periodontal tissues?

Therefore, there is a need for an objective indicator to determine the present inflammatory state of the periodontal tissue. Advanced glycation end products (AGE) are the end products of nonenzymatic glycation and related to cell degradation, cytokine induction, and inflammatory reactions ${ }^{8}$. They interact with several cellular receptors, with the receptor for advanced glycation end products (RAGE) being the most representative'. The bond between AGE and RAGE is associated with various systemic inflammatory processes and also the upregulation of TNF-a, IL-6, and IL-1. The upregulation can cause immune dysfunction, cellular stress, cytokine imbalance, and the progression of periodontitis. RAGEs, when detached from the cell membrane, are called the soluble forms of RAGE (sRAGE). sRAGE could be found in the blood and various other body fluids, and they act as a "decoy receptor" for RAGE or "RAGE inhibitors". In other words, sRAGE can combine with AGE to inhibit the ligand-RAGE interaction between AGE and RAGE ${ }^{10}$. sRAGE can be a potential objective indicator of the current state of periodontitis. In fact, in various inflammatory diseases, the sRAGE value was classified according to the severity ${ }^{11}$. The purpose of this study was to investigate the correlation between SRAGE and the periodontitis status described in several classifications of periodontitis and to confirm if it could be used to determine the current inflammatory state of periodontitis.

\section{Patients and Methods}

\section{Study population}

This retrospective study was performed with the data obtained from 84 patients without any systemic diseases. The 84 participants were recruited from the rheumatology clinic and a section of dentistry at the Seoul National University Bundang Hospital through local advertisements in Bundang and surrounding areas from October 2012 to September 2013. Participants who had undergone periodontal treatment in the past 6 months; were on immunosuppressives, corticosteroids, or antimicrobial therapy which could affect their systemic inflammatory and periodontal conditions, in the past 3 months; or had history of diabetes or inflammatory bowel disease, were excluded. This study was approved by the Institutional Review Board of Seoul National University Bundang Hospital (No. B-1910/570-108).

\section{Clinical and periodontal data collection}

Clinical demographic data such as age, sex, smoking and drinking history, and body mass index (BMI) were recorded. The patients' medical history and history of recent medications were investigated. Various parameters that affect dental health including dental visits within the past 12 months and brushing habits were recorded for all participants.

The periodontal status of all remaining teeth in the oral cavity, except the third molars, was examined by determining the scores for plaque index (PI) and BOP index and measuring probing pocket depths (PPD). The BOP index was determined as the number of BOP points divided by total probing points with each tooth having six probing points (mesiobuccal, buccal, distobuccal, mesiolingual, lingual, and distolingual). PI was similarly determined as the number of tooth surfaces with plaque accumulation divided by the total number of tooth surfaces. The PPD and BOP index scores were measured by a single dentist using a University of North Carolina -15 periodontal probe (Hu Friedy, Chicago, IL, USA). The numbers of remaining teeth were also recorded. All the measurements were performed in accordance with a previously described method ${ }^{12-14}$.

\section{Analysis of blood and gingival crevicular fluid samples}

The levels of a representative bacterium of the 'red complex', Porphyromonas gingivalis, whose numbers increase with the progression of periodontitis, were detected in gingival crevicular fluid (GCF) samples by polymerase chain reaction (PCR). The serum levels of sRAGE and AGE (sAGE) were measured. C-reactive protein (CRP) levels and erythrocyte sedimentation rate (ESR), which are representative blood tests for inflammation, were also determined. Using horseradish peroxidase conjugated to anti-human immunoglobulin $\mathrm{G}(\mathrm{IgG})$ antibodies (1:6,000 dilution; Millipore, Billerica, MA, USA) and 3, 3', 5, 5'-tetramethylbenzidine (Kirkegaard $\&$ Perry Laboratories, Gaithersburg, MD, USA), levels of $P$. gingivalis $\mathrm{IgG}$ antibodies were detected in blood samples. 


\section{Classification of periodontitis}

The most common and easy to use classification of periodontitis was proposed by the American Academy of Periodontology/Centers for Disease Control and Prevention (AAP/CDC) in 2012 based on the National Health and Nutrition Examination Survey data. According to the classification, mild, moderate, and severe forms of periodontitis are determined using PPD and clinical attachment loss $(\mathrm{CAL})^{15}$. In addition, residual PPD of $5 \mathrm{~mm}$, which increases the risk of tooth loss during supportive periodontal therapy for periodontitis, was used as a criterion for periodontitis (PPDs of $<5 \mathrm{~mm}$ at all locations: no/mild periodontitis, PPDs of $\geq 5 \mathrm{~mm}$ in at least one location: moderate periodontitis; PPD5 $)^{16,17}$. Other classifications, proposed by Baelum and López $^{18}$ (López) involving the criteria of $\mathrm{CAL} \geq 4 \mathrm{~mm}$ and
$\mathrm{BOP}$, which indicates the presence of periodontitis, were also used for analysis.

\section{Statistical analysis}

The data obtained from 76 of the 84 participants were statistically analyzed. Data from patients with missing values were excluded. According to the severity of periodontitis, demographic data, data obtained from blood samples and dental examinations, the presence of $P$. gingivalis in GCF, sAGE and sRAGE, and sAGE/sRAGE ratios were analyzed. In each group, continuous variables were compared using the Wilcoxon's rank-sum test, and categorical variables were analyzed with the chi-square test and Fisher's exact test.

The correlations among sRAGE, sAGE, periodontal indices, and inflammation-related variables were assessed by

Table 1. Baseline characteristics, study population stratified by the classification (PPD $5 \mathrm{~mm}$ ) of periodontitis

\begin{tabular}{|c|c|c|c|c|}
\hline \multirow{3}{*}{ Characteristic } & \multirow{3}{*}{ Total $(n=76)$} & \multicolumn{3}{|c|}{ Classification of periodontitis } \\
\hline & & \multicolumn{2}{|c|}{ PPD5 $^{1}$} & \multirow{2}{*}{$P$-value } \\
\hline & & $\mathrm{PPD}<5 \mathrm{~mm}$ & $\mathrm{PPD} \geq 5 \mathrm{~mm}$ & \\
\hline Sex & & & & 0.08 \\
\hline Male & $65(85.5)$ & $38(79.2)$ & 27 (96.4) & \\
\hline Female & $11(14.5)$ & $10(20.8)$ & $1(3.6)$ & \\
\hline Age (yr) & $35.5[30.0-43.5]$ & $32.0[27.5-41.0]$ & $40.0[33.5-46.0]$ & $<0.01$ \\
\hline BMI $\left(\mathrm{kg} / \mathrm{m}^{2}\right)$ & $23.7[22.4-25.6]$ & $23.0[22.4-24.8]$ & $24.0[22.6-26.3]$ & 0.22 \\
\hline Smoking & & & & 0.95 \\
\hline Never & $48(63.2)$ & $30(62.5)$ & $18(64.3)$ & \\
\hline Ever & $15(19.7)$ & $10(20.8)$ & $5(17.9)$ & \\
\hline Current & $13(17.1)$ & 8 (16.7) & $5(17.9)$ & \\
\hline Alcohol & & & & 0.12 \\
\hline Never & $22(28.9)$ & $17(35.4)$ & $5(17.9)$ & \\
\hline Ever & $31(40.8)$ & $20(41.7)$ & $11(39.3)$ & \\
\hline Current & $23(30.3)$ & $11(22.9)$ & $12(42.9)$ & \\
\hline CRP (mg/L) & $0.0[0.0-0.1]$ & $0.0[0.0-0.1]$ & $0.1[0.0-0.1]$ & 0.15 \\
\hline ESR (mm/hr) & $4[2.0-8.0]$ & $3.0[2.0-6.0]$ & $6.0[3.0-10.0]$ & 0.02 \\
\hline Brushing, times/day & & & & 0.72 \\
\hline$>3$ & $47(61.8)$ & $30(62.5)$ & $17(60.7)$ & \\
\hline 2 & $28(36.8)$ & $17(35.4)$ & $11(39.3)$ & \\
\hline $0-1$ & $1(1.3)$ & $1(2.1)$ & 0 & \\
\hline Dental clinic visits in the past $12 \mathrm{mo}$ & & & & 0.93 \\
\hline No & $48(63.2)$ & $31(64.6)$ & $17(60.7)$ & \\
\hline Yes & $28(36.8)$ & $17(35.4)$ & $11(39.3)$ & \\
\hline No. of remaining teeth & $28.0[26.5-28.0]$ & $28.0[27.0-28.0]$ & $28.0[25.5-28.0]$ & 0.19 \\
\hline Mean PPD (mm) & $2.6[2.4-2.8]$ & $2.4[2.3-2.6]$ & $2.9[2.7-3.1]$ & $<0.001$ \\
\hline Mean CAL (mm) & $2.6[2.4-2.9]$ & $2.4[2.3-2.6]$ & 3.0 [2.7-3.3] & $<0.001$ \\
\hline BOP index score $(\%)$ & $9.5[6.2-17.0]$ & $8.3[5.4-11.8]$ & $15.7[9.5-23.6]$ & $<0.001$ \\
\hline Plaque index score (\%) & $26.2[11.1-36.6]$ & $15.6[8.9-33.0]$ & $34.2[19.7-43.8]$ & $<0.01$ \\
\hline$P$. gingivalis antibody level & $2,878.9[1,241.8-5,944.9]$ & $1,737.9[1,093.0-5,116.4]$ & $3,275.8[2,432.4-6,937.1]$ & 0.04 \\
\hline$P$. gingivalis detection by PCR & & & & $<0.001$ \\
\hline No & $43(56.6)$ & $35(72.9)$ & $8(28.6)$ & \\
\hline Yes & $33(43.4)$ & $13(27.1)$ & $20(71.4)$ & \\
\hline
\end{tabular}

(PPD: probing pocket depth, BMI: body mass index, CRP: C-reactive protein, ESR: erythrocyte sedimentation rate, CAL: clinical attachment loss, BOP: bleeding on probing, $P$. gingivalis: Porphyromonas gingivalis, PCR: polymerase chain reaction)

Values are presented as number (\%) or median [interquartile range].

${ }^{1}$ PPDs of $<5 \mathrm{~mm}$ at all locations: no/mild periodontitis, PPDs of $\geq 5 \mathrm{~mm}$ in at least one location: moderate periodontitis.

Keun-Suh Kim et al: Association between soluble forms of the receptor for advanced glycation end products and periodontal disease: a retrospective study. J Korean Assoc Oral Maxillofac Surg 2021 


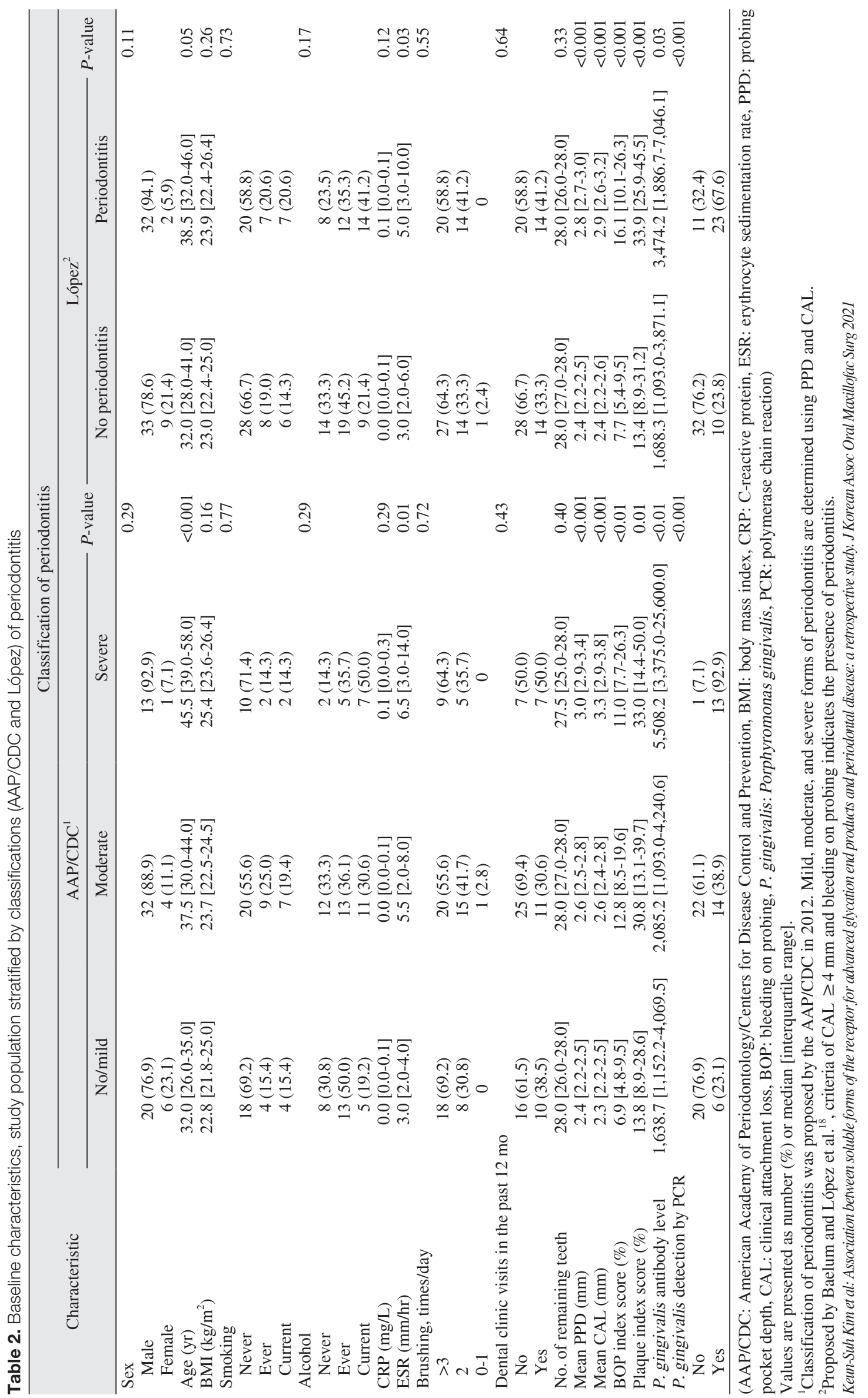




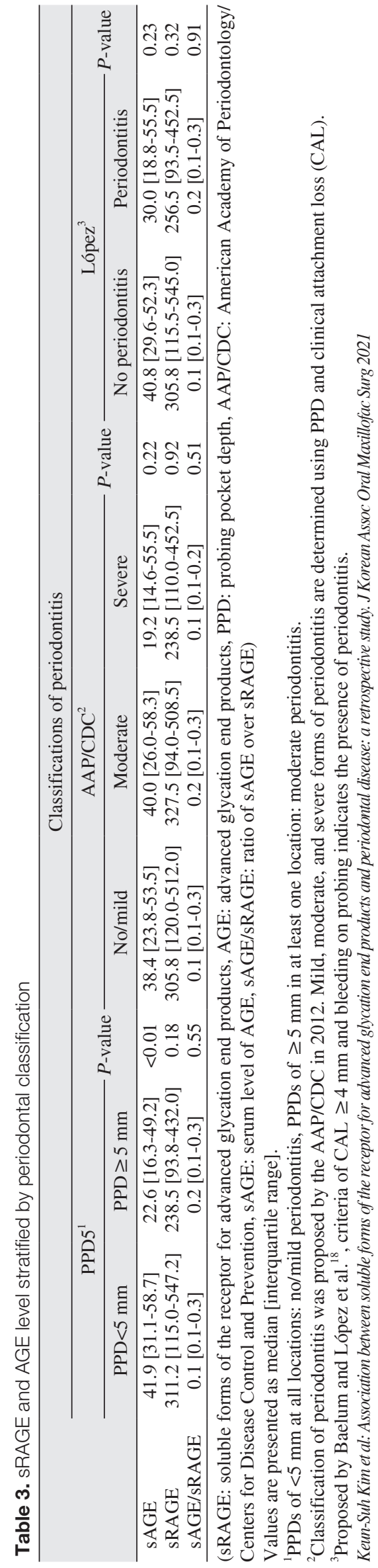

Spearman's rank correlation coefficients. In addition, partial correlation analysis was also performed on variables exhibiting significant changes in correlation coefficients according to the severity of periodontitis. $P<0.05$ was considered statistically significant. Continuous variables were expressed as a median with interquartile range, and categorical variables were presented as a count or percentage. Statistical analysis was performed using the R ver. 3.6.1 (https://www.R-project. org; The R Foundation for Statistical Computing, Vienna, Austria).

\section{Results}

The demographic characteristics of the study population are shown in Table 1. ESR, mean PPD, BOP index scores, PI scores, serum $P$. gingivalis antibody level, and presence of $P$. gingivalis in GCF were significantly different among the groups according to the classifications. There were no statistically significant differences in sex, BMI, CRP level, smoking and drinking history, dental clinic visits in the past 12 months, tooth brushing habits, and number of remaining teeth.

According to the classifications, the mean PPD, BOP index, $P$. gingivalis antibody levels, detection of $P$. gingivalis by PCR, and PI were significantly higher in the study population with periodontitis or more severe periodontitis. This revealed an accurate representation of the periodontal condition by the classifications used in this study.(Tables 1,2)

Table 3 shows the sAGE, sRAGE, and sAGE/sRAGE ratio according to the periodontal status. According to the PPD5 classification, the group with severe periodontitis $(\mathrm{PPD} \geq 5$ $\mathrm{mm}$ ) exhibited lower sAGE and sRAGE levels and a higher sAGE/sRAGE ratio compared to the group without it (PPD $<5$ $\mathrm{mm}$ ), but the differences were not statistically significant except for serum AGE. In the AAP/CDC classification, the sRAGE levels tended to be lower when the severity of periodontitis increased. However, this finding was also not statistically significant. Other values also showed no significant difference. In the classification by López, a similar pattern to the results of the PPD5 classification was shown, but it was not statistically significant.(Fig. 1)

The correlations among sRAGE, sAGE, sAGE/sRAGE ratio, and variables related to periodontitis and systemic inflammatory conditions are shown in Fig. 2. Overall, the correlations were weak. BOP index exhibited relatively strong and negative correlations with sRAGE $(\rho=0.20)$. Different results can be obtained by analyzing the correlation between 

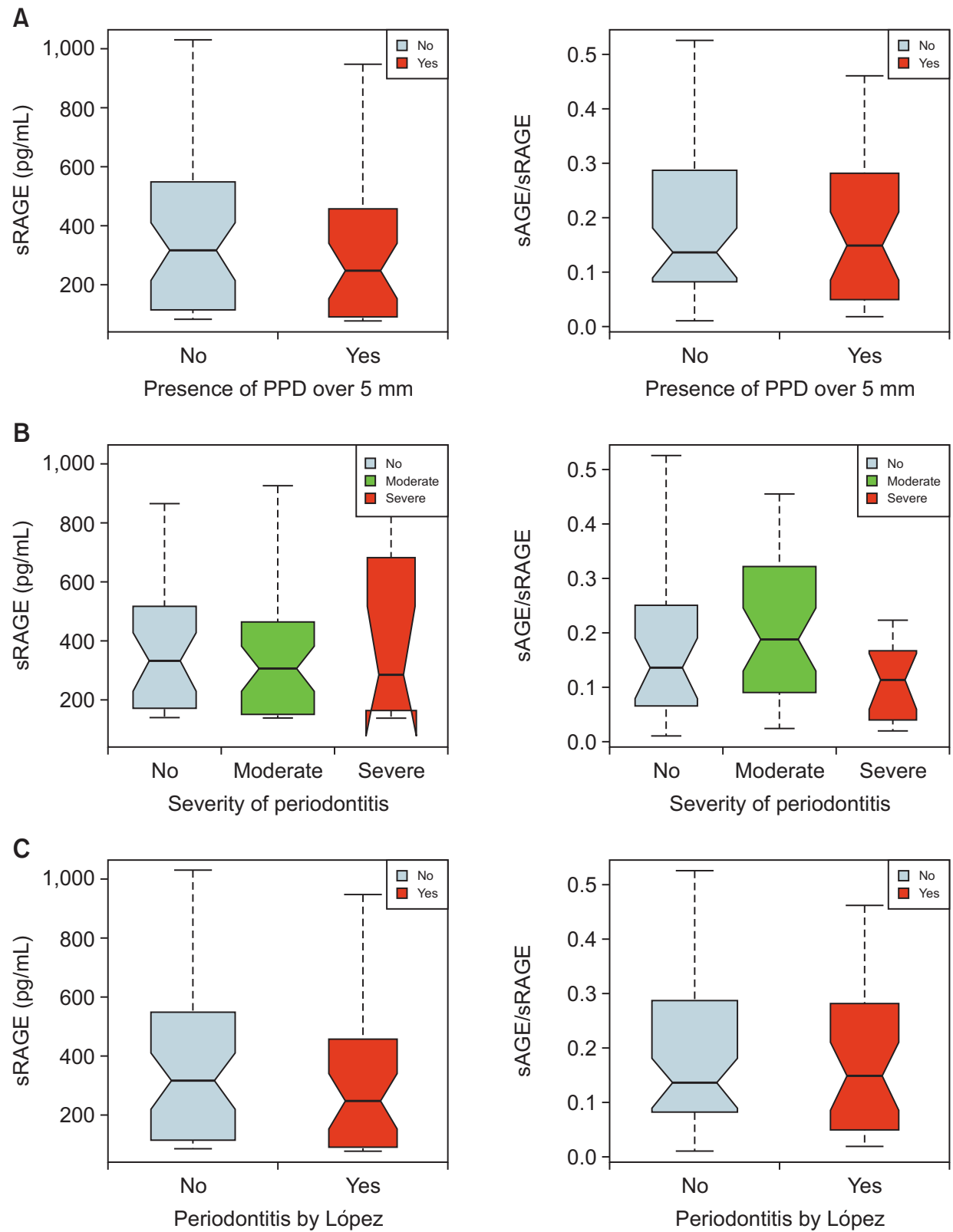

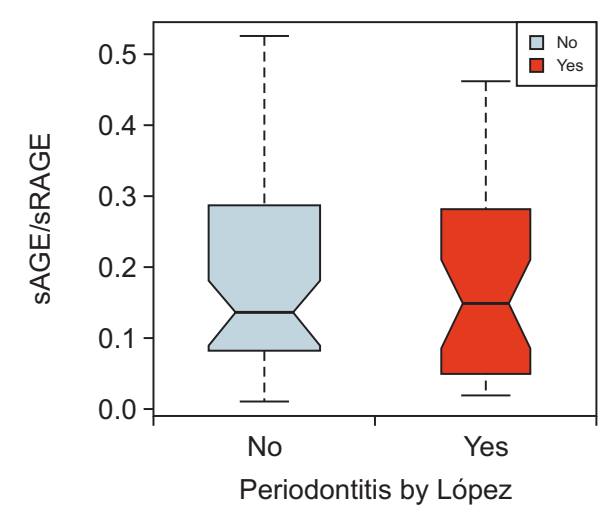

Fig. 1. Boxplot showing the sRAGE level stratified by the classifications of periodontitis. A. PPD5. B. AAP/CDC. C. López. Refer to Table 3 for the definition of PPD5, AAP/CDC, and López. (sRAGE: soluble forms of the receptor for advanced glycation end products, sAGE: serum level of advanced glycation end products, PPD: probing pocket depth, AAP/CDC: American Academy of Periodontology/Centers for Disease Control and Prevention)

Keun-Suh Kim et al: Association between soluble forms of the receptor for advanced glycation end products and periodontal disease: a retrospective study. J Korean Assoc Oral Maxillofac Surg 2021 the BOP index and sRAGE according to the severity or presence of periodontitis. In the $\mathrm{PPD} \geq 5 \mathrm{~mm}$ group, the correlation coefficient of BOP index and sRAGE increased $(\rho=0.20 \rightarrow$ -0.23 ). In contrast, in the $\mathrm{PPD}<5 \mathrm{~mm}$ group, the correlation coefficients between the BOP index and sRAGE decreased $(\rho=0.20 \rightarrow-0.08)$. In the periodontitis group of the López classification, the correlation coefficient of the BOP index and sRAGE also demonstrated a stronger negative correlation compared to the no-periodontitis group $(\rho=0.40$ vs $<0.01)$. In relation to the AAP/CDC classification, as the severity of periodontitis increased, the correlation coefficient between the BOP index and sRAGE exhibited the strongest negative correlation ( $\rho=0.15$ vs -0.21 vs -0.50$)$.(Fig. 2 )
As a result of the partial correlation analysis, which controlled other variables that may affect the sRAGE or BOP index, a stronger negative correlation was found between sRAGE and the BOP index. In each classification, the values of the correlation coefficient were $-0.36,-0.55$, and -0.45 ( $\mathrm{PPD} \geq 5 \mathrm{~mm}$ group, severe group of $\mathrm{AAP} / \mathrm{CDC}$, periodontitis group of López, respectively) in cases with severe periodontitis or exhibiting the presence of periodontitis. Conversely, the ratio of $\mathrm{sAGE} / \mathrm{sRAGE}$ showed a strong positive correlation $(\rho=0.36,0.62,0.45) .($ Table 4$)$ 
A

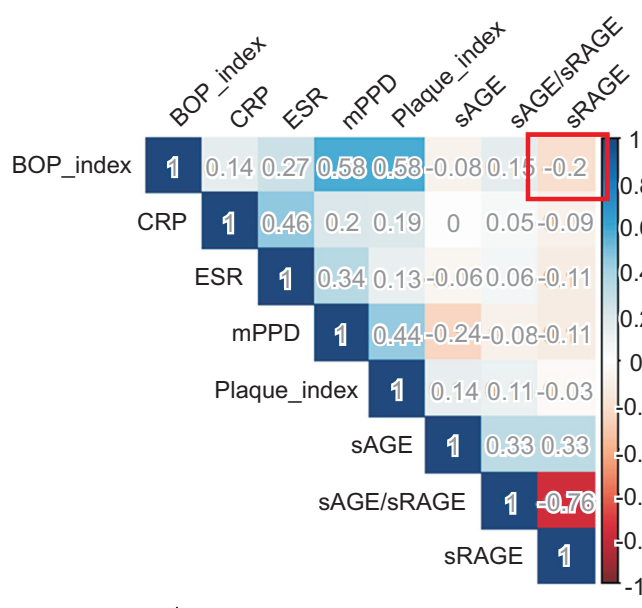

B PPD $<5 \mathrm{~mm}$ group

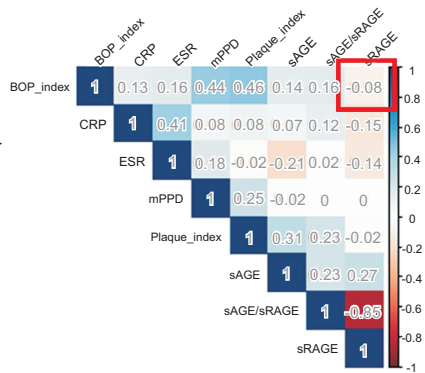

D No periodontitis group

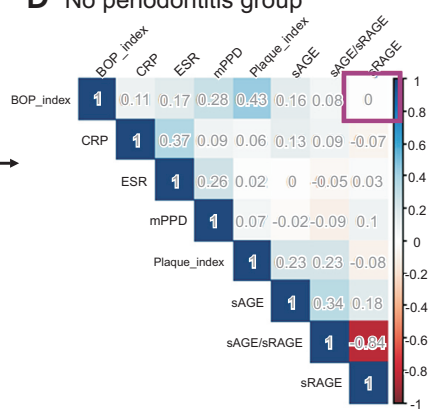

G Moderate periodontitis group

F No/mild periodontitis group
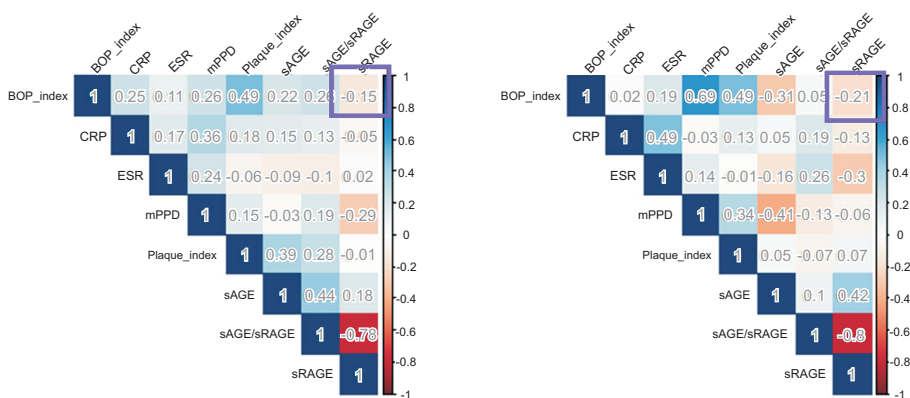

C PPD $\geq 5 \mathrm{~mm}$ group

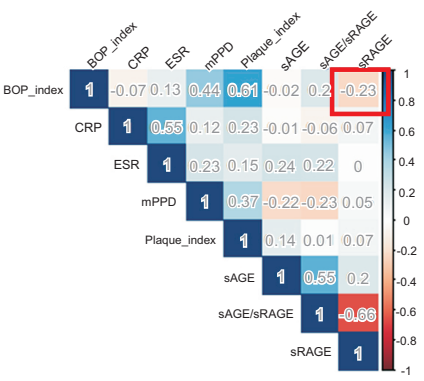

E Periodontitis group

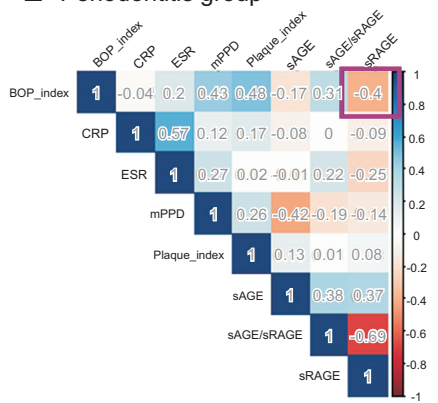

H Severe periodontitis group

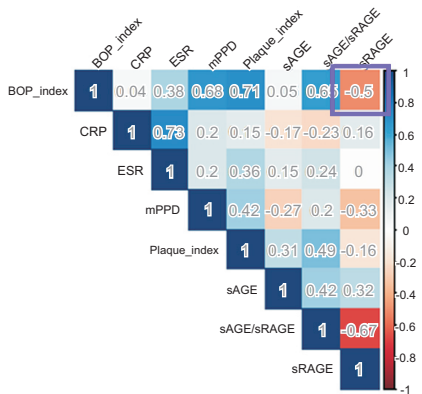

Fig. 2. Correlation among sRAGE, sAGE, SAGE/sRAGE ratio, periodontal indices, and inflammation-related variables displayed by hitmap. A. All patients. B, C. PPD5. D, E. AAP/CDC. F-H. López. Refer to Table 3 for the definition of PPD5, AAP/CDC, and López. (BOP_index: index of bleeding on probing, CRP: C-reactive protein, ESR: erythrocyte sedimentation rate, mPPD: mean probing pocket depth, Plaque index: index of plaque accumulation on teeth, SAGE: serum level of advanced glycation end products, sRAGE: soluble forms of the receptor for AGE, sAGE/sRAGE: ratio of sAGE over sRAGE, PPD: probing pocket depth, AAP/CDC: American Academy of Periodontology/ Centers for Disease Control and Prevention)

Keun-Suh Kim et al: Association between soluble forms of the receptor for advanced glycation end products and periodontal disease: a retrospective study. J Korean Assoc Oral Maxillofac Surg 2021

\section{Discussion}

We studied the correlation between sRAGE and periodontitis in clinical settings. In the various classifications of periodontitis, it was found that sRAGE exhibited a relatively stronger correlation with the current state if periodontitis was present or had worsened.

Most studies on sRAGE involved animal or cell experiments, and few studies have attempted to identify the trends in humans. Although the results of this study were not statistically significant, we observed the tendency for low sRAGE levels in patients with periodontitis. This result is similar to that of a previous study ${ }^{19}$ which analyzed the sRAGE levels in periodontitis patients. Several studies have shown that considering the roles of the AGE-RAGE axis and $\mathrm{SRAGE}^{20}$, it is difficult to predict the state of a disease simply by sRAGE or AGE alone. Initially, sRAGE competes with RAGE and acts as a false receptor for AGE. Therefore, in cases with low serum sRAGE levels, some AGE persists and binds to RAGE. However, in cases with high serum sRAGE levels, lesser amounts of AGE persist, preventing disease development. From this point of view, the values of the sAGE/sRAGE ratio, which represents the relative AGE and sRAGE amounts, were compared according to the severity of periodontitis, but 
Table 4. Partial correlation coefficient

\begin{tabular}{|c|c|c|c|c|c|c|c|c|}
\hline & \multicolumn{8}{|c|}{ Classifications of periodontitis } \\
\hline & \multirow{2}{*}{$\begin{array}{c}\text { Total } \\
(\mathrm{n}=73)\end{array}$} & \multicolumn{2}{|c|}{ PPD5 } & \multicolumn{3}{|c|}{$\mathrm{AAP} / \mathrm{CDC}$} & \multicolumn{2}{|c|}{ López } \\
\hline & & $\mathrm{PPD}<5 \mathrm{~mm}$ & $\mathrm{PPD} \geq 5 \mathrm{~mm}$ & No/mild & Moderate & Severe & No periodontitis & Periodontitis \\
\hline sRAGE and BOP index $^{1}$ & -0.18 & -0.09 & $-0.36^{*}$ & -0.14 & -0.23 & $-0.55^{*}$ & -0.01 & $-0.45 * *$ \\
\hline sAGE/sRAGE and BOP index ${ }^{2}$ & 0.18 & 0.09 & $0.36^{*}$ & 0.13 & 0.18 & $0.62 * *$ & 0.01 & $0.45 * *$ \\
\hline
\end{tabular}

(PPD: probing pocket depth, AAP/CDC: American Academy of Periodontology/Centers for Disease Control and Prevention, sRAGE: soluble forms of the receptor for advanced glycation end products [AGE], BOP: bleeding on probing, sAGE/sRAGE: ratio of serum level of AGE over sRAGE)

Refer to Table 3 for the definition of PPD5, AAP/CDC, and López.

${ }^{1}$ Adjusted for erythrocyte sedimentation rate (ESR), Plaque index, sAGE, mean PPD (mPPD).

${ }^{2}$ Adjusted for ESR, Plaque index, mPPD adjusted.

$* P<0.10 ; * * P<0.05$ statistically significant.

Keun-Suh Kim et al: Association between soluble forms of the receptor for advanced glycation end products and periodontal disease: a retrospective study. J Korean Assoc Oral Maxillofac Surg 2021

no significant results were obtained. The limitation of this study is that the total sample size was small and the number of patients were not matched for each group thereby causing statistically insignificant results.

In the case of correlation analysis among sAGE, sRAGE, sAGE/sRAGE ratio, and indices frequently used as a diagnostic standard for periodontitis, the correlation coefficients were low. However, when the analysis was centered on the correlation between the BOP index, which can indicate the current inflammatory state of the periodontal tissue, and sRAGE, a stronger correlation was found. In a periodontal pocket caused by the loss of alveolar bone, there is increased colonization of anaerobic bacteria and activation of the inflammatory reaction around the periodontal tissue, thereby causing bleeding during probing. In the $\mathrm{PPD} \geq 5 \mathrm{~mm}, \mathrm{AAP} /$ $\mathrm{CDC}$, and López classifications, it was confirmed that the BOP index was significantly higher for more severe disease, and the weak negative correlation between BOP index and sRAGE gradually changed $(\rho=-0.20 \rightarrow-0.23$ or -0.50 or -0.40 ). This indicates that sRAGE can explain the current state of periodontitis. In particular, a stronger negative correlation in the partial correlation coefficient controlling variables indicative of other inflammation or variables that may affect sRAGE supports this result. Additionally, the results of the partial correlation coefficient analysis found that the sAGE/sRAGE ratio and BOP index exhibited a stronger positive correlation than before in the group with severe periodontal disease. This seems to be related to the results of previous studies in which the sAGE/sRAGE ratio was high in the group with severe periodontal disease ${ }^{20}$.

In this paper, we did not have a sufficient number of subjects, and there was a limitation in statistical analysis due to the difference in the ratio of subjects with periodontal disease and subjects without periodontal disease. In addition, it was a healthy patient group and it was confirmed that there were no diabetes or inflammatory bowel disease that could affect sRAGE or sAGE. However, other inflammatory diseases or factors that may contribute to temporary inflammatory conditions were not verified.

\section{Conclusion}

The correlation coefficient between sRAGE and BOP index, which indicates the current inflammatory state of periodontitis, demonstrates a direction consistent with the pathological causal relationship. sRAGE is expected to help determine the current status of periodontitis and its severity. Additional studies with large sample sizes are necessary.

\section{ORCID}

Keun-Suh Kim, https://orcid.org/0000-0002-5986-4810

Yun Jong Lee, https://orcid.org/0000-0001-7615-8611

Soyeon Ahn, https://orcid.org/0000-0003-3440-2027

Yoon-Seok Chang, https://orcid.org/0000-0003-3157-0447

Yonghoon Choi, https://orcid.org/0000-0002-1331-969X

Hyo-Jung Lee, https://orcid.org/0000-0002-0439-7389

\section{Authors' Contributions}

K.S.K., H.J.L., and Y.J.L. participated in conception and design. K.S.K., Y.C., and Y.S.C. participated in administrative support. H.J.L., S.A., and Y.J.L. participated in provision of study materials or patients. K.S.K., H.J.L., and Y.J.L. participated in collection and assembly of data. All authors participated in data analysis, interpretation, manuscript writing, and final approval of manuscript. 


\section{Acknowledgements}

This study was selected in the 3rd Clinical Research Methodology Workshop 2014 of Seoul National University Bundang Hospital (SNUBH) and was supported by a grant from the SNUBH Research Fund (No. 02-2017-039).

\section{Ethics Approval and Consent to Participate}

The authors are accountable for all aspects of the work in ensuring that questions related to the accuracy or integrity of any part of the work are appropriately investigated and resolved. The study was conducted in accordance with the Declaration of Helsinki (as revised in 2013) and approved by the Institutional Review Board of Seoul National University Bundang Hospital (No. B-1910/570-108) and individual consents for this retrospective analysis were waived.

\section{Conflict of Interest}

No potential conflict of interest relevant to this article was reported.

\section{References}

1. GBD 2015 Mortality and Causes of Death Collaborators. Global, regional, and national life expectancy, all-cause mortality, and cause-specific mortality for 249 causes of death, 1980-2015: a systematic analysis for the Global Burden of Disease Study 2015. Lancet 2016;388:1459-544. https://doi.org/10.1016/S01406736(16)31012-1

2. Eke PI, Borgnakke WS, Genco RJ. Recent epidemiologic trends in periodontitis in the USA. Periodontol 2000 2020;82:257-67. https://doi.org/10.1111/prd.12323

3. National Health Insurance Service. 2018 National health screening statistical yearbook. Wonju: National Health Insurance Service; 2019.

4. Jepsen S, Blanco J, Buchalla W, Carvalho JC, Dietrich T, Dörfer C, et al. Prevention and control of dental caries and periodontal diseases at individual and population level: consensus report of group 3 of joint EFP/ORCA workshop on the boundaries between caries and periodontal diseases. J Clin Periodontol 2017;44 Suppl 18:S8593. https://doi.org/10.1111/jcpe.12687

5. Slavkin HC, Baum BJ. Relationship of dental and oral pathology to systemic illness. JAMA 2000;284:1215-7. https://doi.org/10.1001/ jama.284.10.1215

6. Pihlstrom BL, Michalowicz BS, Johnson NW. Periodontal diseases. Lancet 2005;366:1809-20. https://doi.org/10.1016/S01406736(05)67728-8
7. Nair P, Sutherland G, Palmer RM, Wilson RF, Scott DA. Gingival bleeding on probing increases after quitting smoking. J Clin Periodontol 2003;30:435-7. https://doi.org/10.1034/j.1600051x.2003.20039.x

8. Ilea A, Băbțan AM, Boșca BA, Crişan M, Petrescu NB, Collino $\mathrm{M}$, et al. Advanced glycation end products (AGEs) in oral pathology. Arch Oral Biol 2018;93:22-30. https://doi.org/10.1016/ j.archoralbio.2018.05.013

9. Oliveira MIA, de Souza EM, de Oliveira Pedrosa F, Réa RR, da Silva Couto Alves A, Picheth G, et al. RAGE receptor and its soluble isoforms in diabetes mellitus complications. J Bras Patol Med Lab 2013;49:97-108. https://doi.org/10.1590/S167624442013000200004

10. Sakaguchi T, Yan SF, Yan SD, Belov D, Rong LL, Sousa M, et al Central role of RAGE-dependent neointimal expansion in arterial restenosis. J Clin Invest 2003;111:959-72. https://doi.org/10.1172/ JCI17115

11. Yan SF, Ramasamy R, Schmidt AM. Soluble RAGE: therapy and biomarker in unraveling the RAGE axis in chronic disease and aging. Biochem Pharmacol 2010;79:1379-86. https://doi.org/10.1016/ j.bcp.2010.01.013

12. Page RC, Eke PI. Case definitions for use in population-based surveillance of periodontitis. J Periodontol 2007;78(7 Suppl):1387-99. https://doi.org/10.1902/jop.2007.060264

13. O'Leary TJ, Drake RB, Naylor JE. The plaque control record. J Periodontol 1972;43:38. https://doi.org/10.1902/jop.1972.43.1.38

14. Ainamo J, Bay I. Problems and proposals for recording gingivitis and plaque. Int Dent J 1975;25:229-35.

15. Eke PI, Page RC, Wei L, Thornton-Evans G, Genco RJ. Update of the case definitions for population-based surveillance of periodontitis. J Periodontol 2012;83:1449-54. https://doi.org/10.1902/ jop.2012.110664

16. McCracken G, Asuni A, Ritchie M, Vernazza C, Heasman P. Failing to meet the goals of periodontal recall programs. What next? Periodontol 2000 2017;75:330-52. https://doi.org/10.1111/ prd.12159

17. Matuliene G, Pjetursson BE, Salvi GE, Schmidlin K, Brägger U, Zwahlen M, et al. Influence of residual pockets on progression of periodontitis and tooth loss: results after 11 years of maintenance. J Clin Periodontol 2008;35:685-95. https://doi.org/10.1111/j.1600051X.2008.01245.x

18. Baelum V, López R. Defining a periodontitis case: analysis of a never-treated adult population. J Clin Periodontol 2012;39:10-9. https://doi.org/10.1111/j.1600-051X.2011.01812.x

19. Detzen L, Cheng B, Chen CY, Papapanou PN, Lalla E. Soluble forms of the receptor for advanced glycation endproducts (RAGE) in periodontitis. Sci Rep 2019;9:8170. https://doi.org/10.1038/ s41598-019-44608-2

20. Prasad K. Is there any evidence that AGE/sRAGE is a universal biomarker/risk marker for diseases? Mol Cell Biochem 2019;451: 139-44. https://doi.org/10.1007/s11010-018-3400-2

How to cite this article: Kim KS, Lee YJ, Ahn S, Chang YS, Choi Y, Lee HJ. Association between soluble forms of the receptor for advanced glycation end products and periodontal disease: a retrospective study. J Korean Assoc Oral Maxillofac Surg 2021;47:445-453. https://doi.org/10.5125/jkaoms.2021.47.6.445 